\title{
Immunogenicity and protective efficacy of Salmonella enterica serovar Pullorum pathogenicity island 2 mutant as a live attenuated vaccine candidate
}

\author{
Junlei Yin, Zhao Cheng, Lijuan Xu, Qiuchun Li, Shizhong Geng, Zhiming Pan and Xinan Jiao*
}

\begin{abstract}
Background: Salmonella enterica serovar Pullorum (S. Pullorum) causes Pullorum disease (PD), a severe systemic disease of poultry and results in considerable economic losses in developing countries. In order to develop a safe and immunogenic vaccine, the immunogenicity and protective efficacy of S06004 $\triangle$ SPI2, a Salmonella pathogenicity island 2 (SPI2) deleted mutant of S. Pullorum was evaluated in 2-day old chickens.

Results: Single intramuscular vaccination with S06004 $\triangle$ SPI2 $\left(2 \times 10^{7} \mathrm{CFU}\right)$ of chickens revealed no differences in body weight or clinical symptoms compared to control group. S06004 $\triangle$ SPI2 bacteria can colonize and persistent in liver and spleen of vaccinated chickens approximately 14 days, and specific humoral and cellular immune responses were significantly induced. Vaccination of chickens offered efficient protection against S. Pullorum strain S06004 and S. Gallinarum strain SG9 challenge, respectively, at 10 days post vaccination (dpv) based on mortality and clinical symptoms compared to control group.
\end{abstract}

Conclusions: These findings suggest that S06004 $\triangle$ SPI2 appears to be a highly immunogenic and efficient live attenuated vaccine candidate.

Keywords: Salmonella enterica serovar Pullorum, Pullorum disease, Salmonella pathogenicity island 2, live attenuated vaccine

\section{Background}

Salmonella enterica serovar Pullorum (S. Pullorum) is the causative agent of Pullorum disease (PD), an acute systemic disease that results in high morbidity and mortality in young chicks and a loss of weight, decreased fertility and hatchability, lesions, diarrhea and abnormalities of the reproductive tract in infected adults, it can be transmitted vertically to chicks through eggs [1]. This disease remains a big threat of restricting the growth of the poultry industry in developing countries [2]. As a close relative of S. Pullorum, Salmonella enterica serovar Gallinarum (S. Gallinarum) causes Fowl typhoid (FT), a severe

\footnotetext{
* Correspondence: jiao@yzu.edu.cn

Jiangsu Key Laboratory of Zoonosis, Jiangsu Co-innovation Center for Prevention and Control of Important Animal Infectious Diseases and
} Zoonoses, Yangzhou University, Yangzhou, Jiangsu 225009, P R China systemic disease with significant morbidity and mortality in poultry in many countries [2-5].

Vaccination is an effective strategy for the control of Salmonella infections, both humoral and cellular immunity are required for ideal Salmonella vaccines [6]. Live vaccines offer greater protection than killed vaccines because higher cellular immune response could be induced, it is important for clearance of Salmonella infections [6].

As an indispensable virulence determinant associated with the systemic infections, Salmonella pathogenicity island 2 (SPI2) can encode type III secretion system 2 (T3SS2), which is induced after invasion, and the T3SS2 secreted effectors are essential for Salmonella to survive and replicate inside various cell types $[7,8]$. There are some papers on the vaccine potential of $S$. Enteritidis, $S$. Typhimurium and $S$. Typhi mutants with deletion of SPI2 or other 
key genes located within the pathogenicity island display decreased virulence in poultry, pigs, cattle, mice, and humans [9-14]. Therefore, in order to determine whether the SPI2 mutant strain of $S$. Pullorum has the vaccine potential, we evaluated the immunogenicity and protective efficacy of S06004 $\Delta$ SPI2 in susceptible HY-line white chickens. Our results showed that intramuscular vaccination with S06004 $\Delta$ SPI2 provides efficient protection against challenges with $S$. Pullorum and S. Gallinarum.

\section{Methods}

\section{Experimental animals}

The animal experiments were conducted with the approval of the Animal Care and Ethics Committee of Yangzhou University. HY-line white chicken eggs were hatched and the chickens were detected for freedom from any clinical signs of enteric disease and negative for Salmonella. Two-day old chickens were used in this study and given antibiotic-free food and water throughout the experimental period.

\section{Bacterial strains}

S. Pullorum S06004 (accession No. CP006575.1), a nalidixic acid-resistant $\left(\mathrm{Nal}^{\mathrm{r}}\right)$ clinical isolate obtained from chickens with Pullorum disease in the Jiangsu Province of China in 2006 [15], and the virulent wild type $S$. Gallinarum strain SG9 $\left(\mathrm{Nal}^{\mathrm{r}}\right)$, supplied by Dr. Barrow [16], were used as challenge strains. S06004 $\triangle$ SPI2 $\left(\mathrm{Nal}^{\mathrm{r}}\right.$, the whole SPI2 $(\sim 40 \mathrm{~kb})$ deleted mutant of $S$. Pullorum S06004), constructed using the one-step inactivation method described by Datsenko and Wanner [17, 18], was used as the vaccine candidate for this study. Bacterial strains were stored as frozen cultures in Luria-Bertani (LB) broth with $20 \%$ glycerol at $-70{ }^{\circ} \mathrm{C}$ before use. LB broth, LB solid (15 g/L agar) and XLT4 (Difco) agar were used for culturing bacteria at $37{ }^{\circ} \mathrm{C}$. The media were supplemented with $\mathrm{Nal}(40 \mu \mathrm{g} / \mathrm{ml})$ as required.

\section{Bacterial inoculation in chickens}

One hundred 2-day old chickens were randomly assigned to 2 groups: vaccinated group $(n=45)$ and control group $(n=55)$. The vaccinated group was intramuscularly immunized with $2 \times 10^{7}$ CFU S06004 $\triangle$ SPI2 in $100 \mu \mathrm{l}$ phosphate buffered saline (PBS), while control group was unimmunized and only received equal amounts PBS.

\section{Changes of body weight and clinical symptoms after vaccination}

Body weights of these chickens were measured at 5, 12 and 19 days post vaccination (dpv), and they were monitored for 19 days for clinical signs of disease, which included anorexia, diarrhea and depression, etc.
Bacterial persistence and clearance from internal organs Liver and spleen samples of five chickens from each group were aseptically collected at 5, 7, 10, 14 and $21 \mathrm{dpv}$ for bacterial recovery. Then they were weighed and suspended in $1 \mathrm{ml}$ PBS and homogenized individually. Homogenates $(100 \mu \mathrm{l})$ of different dilutions were inoculated on XLT4 agar (containing $40 \mu \mathrm{g} / \mathrm{ml} \mathrm{Nal}$ ) for enumeration and incubated for $20 \mathrm{~h}$ at $37^{\circ} \mathrm{C}$. The bacterial number in the sample was counted and expressed as $\log 10 \mathrm{CFU} / \mathrm{g}$, negative samples were indicated as $0 \mathrm{CFU} / \mathrm{g}$.

\section{Immune responses induced by the vaccine strain}

Humoral immune responses were evaluated through determination of Specific antibody IgG levels by Enzymelinked immunosorbent assay (ELISA), using heat-killed whole $S$. Pullorum bacteria as coating antigen as previously described [19]. Serum samples were collected from five chickens of each group at 3, 7, 14 and $21 \mathrm{dpv}$, and diluted 1:50 to be used as the primary antibody. The secondary antibody was Horseradish peroxidase (HRP)conjugated rabbit anti-chicken IgG (1:10,000 dilution). The bound HRP activity was determined using ophenylenediamine dihydrochloride (Sigma), and the $\mathrm{OD}_{492}$ was determined with an ELISA reader after the reactions were stopped by $2 \mathrm{M} \mathrm{H}_{2} \mathrm{SO}_{4}$.

Cellular immune responses were evaluated by the peripheral mononuclear cell proliferation assay as previously described $[20,21]$. Soluble antigen was prepared from the wild type S. Pullorum strain S06004. Peripheral lymphocytes were separated from blood of five birds per group using the Histopaque -1077 (Sigma) at 7, 14 and $21 \mathrm{dpv}$. After trypan blue dye exclusion testing, a viable mononuclear cell suspension $(100 \mu \mathrm{l})$ at $1 \times 10^{6} \mathrm{CFU} / \mathrm{ml}$ in RPMI-1640 medium with $10 \%$ fetal calf serum, $2 \mathrm{mM} \mathrm{L}$-glutamine, $50 \mathrm{U} / \mathrm{ml}$ of penicillin and $50 \mu \mathrm{g} / \mathrm{ml}$ of streptomycin was incubated in triplicate in 96-well tissue culture plates with $50 \mu \mathrm{l}$ of medium alone or medium containing $4 \mu \mathrm{g} / \mathrm{ml}$ of soluble antigen at $41{ }^{\circ} \mathrm{C}$ (in a humidified $5 \% \mathrm{CO}_{2}$ atmosphere for $48 \mathrm{~h}$ ). The proliferation of stimulated lymphocytes was measured using adenosine triphosphate (ATP) bioluminescence with the ViaLight ${ }^{\circ}$ Plus Kit (Lonza Rockland, ME, USA). The blastogenic response against soluble antigen was

Table 1 Mean body weights of chickens after vaccination. The vaccinated group was intramuscularly immunized with $2 \times 10^{7} \mathrm{CFU}$ S06004 $\triangle$ SPI2 in 2-day old chickens, and control group received $100 \mu \mathrm{l}$ PBS

\begin{tabular}{llll}
\hline Group & \multicolumn{3}{l}{ Mean body weight per chicken at dpv $(\mathrm{g})$} \\
\cline { 2 - 4 } & 5 & 12 & 19 \\
\hline vaccinated & $65.416 \pm 0.418$ & $113.878 \pm 0.493$ & $186.583 \pm 0.716$ \\
Control & $64.592 \pm 0.782$ & $114.618 \pm 0.795$ & $187.171 \pm 0.385$
\end{tabular}

There were no significant differences between groups at any time point $(P>0.05)$ 

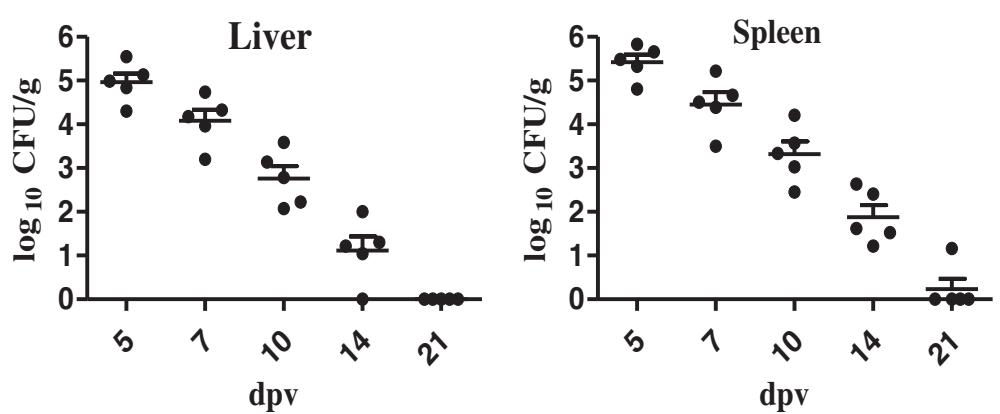

Fig. 1 Bacterial recovery from liver and spleen of the vaccinated chickens. The vaccinated group was intramuscularly immunized with $2 \times 10^{7} \mathrm{CFU}$ S06004 SPI2 in 2-day old chickens, and control group received $100 \mu \mathrm{l}$ PBS. Values represent the mean \pm SEM $\log _{10} \mathrm{CFU} / \mathrm{g}$. All liver and spleen samples of control group were negative

expressed as the mean stimulation index $(\mathrm{SI})$ as previously described [20].

\section{Evaluation of immune protection}

Protective efficacy of S06004 $\Delta$ SPI2 against challenges with S. Pullorum and S. Gallinarum were assessed, based on survival rates and clinical symptoms (including anorexia, diarrhea, depression, high morbidity and mortality). At 10 dpv, twenty chickens from vaccinated group were randomly divided into two groups of 10 animals (group A and $\mathrm{C}$ ), thirty chickens from control group were randomly divided into three groups of 10 animals (group B, D and E). Group A and B were challenged intramuscularly with $2 \times 10^{9}$ CFU S06004 in $100 \mu \mathrm{l}$ of PBS. Groups C and D received equal amounts of SG9. Group E only received $100 \mu \mathrm{l}$ PBS. The surviving birds were counted at 21 days post challenge, and clinical symptoms were recorded every day from 1-35 dpv.

\section{Statistical analysis}

All data were expressed as mean \pm standard error of the mean (SEM) values unless otherwise specified and analyzed with GraphPad Prism. P values less than 0.05 were considered significant when using one-way analysis of variance (ANOVA).

\section{Results}

\section{Changes of body weight and clinical symptoms after vaccination}

After vaccination with S06004 $\triangle$ SPI2, the mean body weight of each chicken in vaccinated group and control group at 5,12 and $19 \mathrm{dpv}$ were shown in Table 1. No significant differences and no clinical signs (anorexia, diarrhea and depression) were observed between the two groups.

\section{Bacterial persistence and clearance in internal organs}

All liver and spleen samples of control group were negative for Salmonella recovery. As shown in Fig. 1, the considerably decreased bacterial counts of vaccinated group were continuously observed through to $21 \mathrm{dpv}$ in both liver and spleen, but S06004 $\Delta$ SPI2 bacteria can colonize and persistent in liver and spleen of vaccinated chickens approximately 14 days. Only one spleen sample was positive and no liver sample was positive at $21 \mathrm{dpv}$.

\section{Humoral and cellular immune responses}

Humoral immune responses were evaluated by measuring specific serum IgG levels at 3, 7, 14 and $21 \mathrm{dpv}$ using ELISA. The mean $\mathrm{OD}_{492}$ values of vaccinated group were $0.221 \pm 0.019, \quad 0.484 \pm 0.039, \quad 0.678 \pm 0.056$ and $1.032 \pm$ 0.064 at $3,7,14$ and $21 \mathrm{dpv}$, respectively (Fig. 2). The chickens in vaccinated group had significantly higher serum IgG levels than those in control group at 7, 14 and $21 \mathrm{dpv}$. The considerably elevated serum IgG levels of vaccinated group were continuously observed through to $21 \mathrm{dpv}$.

Cellular immune responses were examined by the peripheral mononuclear cell proliferation assay. The mean SI values of vaccinated group were $3.124 \pm 0.138,3.495 \pm$ 0.188 and $2.667 \pm 0.189$ at 7,14 and $21 \mathrm{dpv}$, respectively (Fig. 3). All tested chickens in vaccinated group revealed

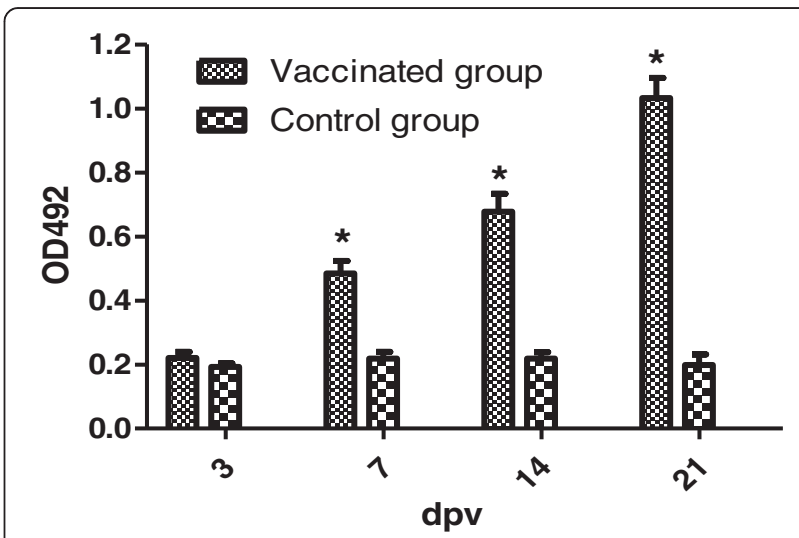

Fig. 2 Determination of serum lgG levels. Vaccinated group and control group refer to Fig. 1. Values represent the mean \pm SEM. * Significant difference compared to the control group, $P<0.05$ 


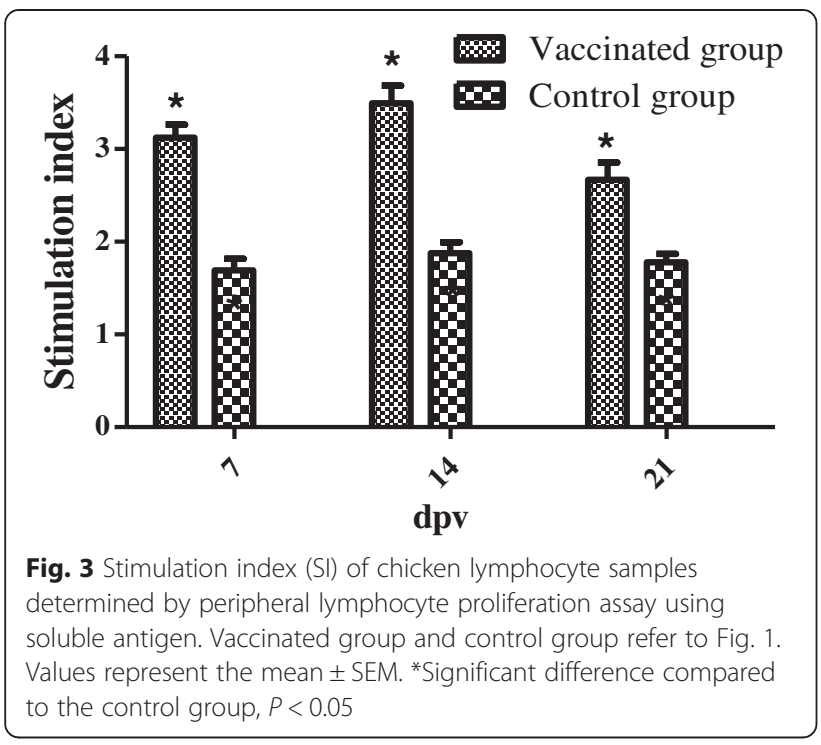

considerably elevated SI values compared to control group, and the significantly elevated SI values was continuously observed at $14 \mathrm{dpv}$, but was reduced at $21 \mathrm{dpv}$.

\section{Immune protection}

The percent survival of chickens which had been vaccinated intramuscularly with $S$. Pullorum mutant S06004 $\triangle$ SPI2 followed by challenge with the parent $S$. Pullorum strain S06004 or S. Gallinarum strain SG9 at $10 \mathrm{dpv}$ was shown in Table 2. One immunized chicken died, whereas nine chickens died in control group B after challenged with S06004. Three immunized chickens died, whereas all ten chickens died in control group D after challenged with SG9. The clinical symptoms (high morbidity and mortality, anorexia, diarrhea, depression) of group $\mathrm{A}$ and $\mathrm{C}$ were slight and temporary after challenged compared to group $\mathrm{E}$, and the chickens had recovered by 3-7 days post challenge; but these clinical symptoms were observed in group B and D. S06004 $\triangle$ SPI2 conferred effective protection.

\section{Discussion}

In this work, we evaluated the immunogenicity and protective efficacy of a Salmonella pathogenicity island 2 (SPI2) deleted mutant of $S$. Pullorum (S06004 $\Delta$ SPI2) to serve as a live vaccine against PD and FT in susceptible HY-line white chickens on the basis of changes of body weight and clinical symptoms, bacterial persistence and clearance, humoral and cellular immune responses, and protective efficiency.

In order to evaluate the effects of S06004 $\triangle$ SPI2 on growth performance in chickens, we recorded the body weight increases and observed the clinical symptoms after intramuscular vaccination. Our results showed that S06004 $\triangle$ SPI2 has almost no side effects on growth performance in chickens. T3SS2 encoded by SPI2 is essential for Salmonella colonization and persistence in host. With the absence of a functional T3SS2, Salmonella is cleared more rapidly than the parental wild type strain from the host, and some studies have failed to isolate SPI2 mutants from liver and spleen after oral inoculation $[5,16,22]$. Here, our results showed that S06004 $\Delta$ SPI2 can colonize and persist in liver and spleen of vaccinated chickens approximately 14 days, this may be related to the breed of chicken, the routes of vaccination and the dose of inoculation.

Specific humoral and cellular immune responses induced by the live attenuated vaccines of Salmonella are crucial for the natural host [6]. To investigate the specific humoral immune responses imparted by the candidate, we examined the specific serum IgG antibody level by indirect ELISA, there was a strong specific serum IgG level in vaccinated chickens, and the antibodies were detectable at 7 dpv. The vaccinated chickens showed significantly elevated IgG levels compared to non-immunized chickens. S. Enteritidis SPI2 mutant can also induce significant increase of antibodies in chickens [9]. Cellular immune responses play a central role in protection against Salmonella challenge, because Salmonella are facultative intracellular pathogens [23]. We further evaluated the cellular immune responses imparted by the candidate in chickens using the peripheral lymphocyte proliferation assay. In the present study, a

Table 2 Protective efficacy of S06004 $\triangle$ SPI2. Group A and C were intramuscularly immunized with $2 \times 10^{7}$ CFU S06004 $\triangle$ SPI2 in 2-day old chickens, group B, D and E were nonimmunized. At 10 dpv, group A-D were challenged

\begin{tabular}{|c|c|c|c|c|c|c|c|}
\hline \multirow[t]{2}{*}{ Group } & \multirow{2}{*}{$\begin{array}{l}\text { Vaccination } \\
\text { Strain }\end{array}$} & \multirow[t]{2}{*}{ Number } & \multicolumn{3}{|c|}{ Challenge } & \multirow[t]{2}{*}{ Survivors/Total } & \multirow[t]{2}{*}{ Survival rate (\% } \\
\hline & & & Strain & Route & Dose (CFU) & & \\
\hline A & S06004 $\triangle S P I 2$ & 10 & S06004 & intramuscularly & $2 \times 10^{9}$ & $9 / 10$ & $90^{*}$ \\
\hline B & PBS & 10 & S06004 & intramuscularly & $2 \times 10^{9}$ & $1 / 10$ & 10 \\
\hline C & S06004 $\triangle S P I 2$ & 10 & SG9 & intramuscularly & $2 \times 10^{9}$ & $7 / 10$ & $70^{*}$ \\
\hline D & PBS & 10 & SG9 & intramuscularly & $2 \times 10^{9}$ & $0 / 10$ & 0 \\
\hline$E$ & PBS & 10 & - & - & - & $10 / 10$ & 100 \\
\hline
\end{tabular}

${ }^{*} P<0.05$ for comparison of group $A$ with group $B$, and group $C$ with group $D$ 
significantly elevated cellular immune response was clearly observed in chickens immunized with S06004 $\Delta$ SPI2, but the significantly elevated SI value was decreased at $21 \mathrm{dpv}$, it is related to the restricted colonization of bacteria in internal organs $[24,25]$. Taken together, the specific humoral and cellular immune responses were clearly observed in the vaccinated chickens in this study.

Several previous reports have shown that live attenuated Salmonella vaccines can confer effective crossprotection to different pathogenic Salmonella serovars $[26,27]$. Here, we evaluated the protective efficacy of the candidate vaccine against challenge intramuscularly with $S$. Pullorum and S. Gallinarum, respectively, based on survival rates and clinical symptoms in HY-line white chickens. The survival rates were $90 \%$ and $70 \%$ following respective challenge with $S$. Pullorum and $S$. Gallinarum in vaccinated chickens; but in the control groups, the survival rates were $10 \%$ and 0 , respectively. The light and temporary clinical symptoms of vaccinated chickens (group A and C) had recovered by 3-7 days post-challenge. Recently, our results also showed that S06004 $\Delta$ SPI2 can be used as a live attenuated oral vaccine [28]. Overall, these results showed that the candidate vaccine S06004 $\Delta$ SPI2 can afford effective protection for acute systemic PD and FT infection.

\section{Conclusions}

Our present work demonstrated that the vaccination of susceptible chickens with the candidate vaccine S06004 $\triangle$ SPI2 conferred development of acquired immunity and efficient protection for the experimental systemic PD and FT infection. Taken together, the SPI2 mutant strain of $S$. Pullorum has the potential of being used as a safe, novel, highly immunogenic vaccine against PD and FT.

\footnotetext{
Abbreviations

S. Pullorum: Salmonella enterica serovar Pullorum; S. Gallinarum: Salmonella enterica serovar Gallinarum; PD: Pullorum disease; FT: Fowl typhoid; SPI2: Salmonella pathogenicity island 2; T3SS2: Type III secretion system 2; Dpv: Days post vaccination; LB: Luria-Bertani; PBS: Phosphate buffered saline; ELISA: Enzyme-linked immunosorbent assay; SI: Stimulation index.
}

\section{Competing interests}

The authors declare that they have no competing interests.

\section{Authors' contributions}

$\mathrm{XJ}, \mathrm{QL}$ and $\mathrm{JY}$ designed the experiments, SG and ZP conducted experiments, $J Y, Z C$ and $L X$ performed the experiments, JY and ZC analyzed the data and drafted the manuscript, $X J$ finalized the manuscript. All authors read and approved the final manuscript.

\section{Acknowledgements}

This work was supported by the Key Program of the National Natural Science Foundation of China (31230070), Colleges and Universities of Jiangsu Province Plans to Graduate Research and Innovation (CXZZ13_0917), National Science \& Technology Pillar Program (2014BAD13B02), National Natural Science Foundation of China (31201905), the Priority Academic Program Development of Jiangsu Higher Education Institutions (PAPD).
Received: 30 March 2015 Accepted: 16 July 2015

Published online: 24 July 2015

\section{References}

1. Rettger LF. Further Studies on fatal Septicemia in Young Chickens, or "White Diarrhea.". J Med Res. 1909;21:115-23.

2. Barrow PA, Freitas Neto OC. Pullorum disease and fowl typhoid-new thoughts on old diseases: a review. Avian Pathol. 2011;40:1-13.

3. Li J, Smith NH, Nelson K, Crichton PB, Old DC, Whittam TS, et al. Evolutionary origin and radiation of the avian-adapted non-motile salmonellae. J Med Microbiol. 1993;38:129-39.

4. Baumler AJ, Tsolis RM, Ficht TA, Adams LG. Evolution of host adaptation in Salmonella enterica. Infect Immun. 1998:66:4579-487.

5. Wigley P, Berchieri Jr A, Page KL, Smith AL, Barrow PA. Salmonella enterica serovar Pullorum persists in splenic macrophages and in the reproductive tract during persistent, disease-free carriage in chickens. Infect Immun. 2001;69:7873-9.

6. Mastroeni P, Chabalgoity JA, Dunstan SJ, Maskell DJ, Dougan G. Salmonella: immune responses and vaccines. Vet J. 2001;161:132-64.

7. Galan JE. Salmonella interactions with host cells: type III secretion at work. Annu Rev Cell Dev Bi. 2001;17:53-86.

8. Waterman SR, Holden DW. Functions and effectors of the Salmonella pathogenicity island 2 type III secretion system. Cell Microbiol. 2003:5:501-11.

9. Matulova M, Havlickova H, Sisak F, Rychlik I. Vaccination of chickens with Salmonella Pathogenicity Island (SPI) 1 and SPI2 defective mutants of Salmonella enterica serovar Enteritidis. Vaccine. 2012;30:2090-7.

10. Khan SA, Stratford R, Wu T, McKelvie N, Bellaby T, Hindle Z, et al. Salmonella typhi and S. typhimurium derivatives harbouring deletions in aromatic biosynthesis and Salmonella Pathogenicity Island-2 (SPI-2) genes as vaccines and vectors. Vaccine. 2003;21:538-48.

11. Boyen F, Pasmans F, Van Immerseel F, Morgan E, Botteldoorn N, Heyndrickx $M$, et al. A limited role for SsrA/B in persistent Salmonella Typhimurium infections in pigs. Vet Microbiol. 2008;128:364-73.

12. Coombes BK, Coburn BA, Potter AA, Gomis S, Mirakhur K, Li Y, et al. Analysis of the contribution of Salmonella pathogenicity islands 1 and 2 to enteric disease progression using a novel bovine ileal loop model and a murine model of infectious enterocolitis. Infect Immun. 2005;73:7161-9.

13. Dieye $Y$, Ameiss K, Mellata M, Curtiss 3rd R. The Salmonella Pathogenicity Island (SPI) 1 contributes more than SPI2 to the colonization of the chicken by Salmonella enterica serovar Typhimurium. BMC Microbiol. 2009;9:3.

14. Karasova D, Sebkova A, Havlickova H, Sisak F, Volf J, Faldyna M, et al. Influence of 5 major Salmonella pathogenicity islands on NK cell depletion in mice infected with Salmonella enterica serovar Enteritidis. BMC Microbiol. 2010;10:75.

15. Geng S, Jiao X, Barrow PA, Pan Z, Chen X. Virulence determinants of Salmonella Gallinarum biovar Pullorum identified by PCR signature-tagged mutagenesis and the spiC mutant as a candidate live attenuated vaccine. Vet Microbiol. 2014;168:388-94.

16. Jones MA, Wigley P, Page KL, Hulme SD, Barrow PA. Salmonella enterica serovar Gallinarum requires the Salmonella pathogenicity island 2 type III secretion system but not the Salmonella pathogenicity island 1 type III secretion system for virulence in chickens. Infect Immun. 2001;69:5471-6.

17. Datsenko KA, Wanner BL. One-step inactivation of chromosomal genes in Escherichia coli K-12 using PCR products. Proc Natl Acad Sci U S A. 2000;97:6640-5.

18. Yin J, Wu Y, Lin Z, Wang $X, \mathrm{Hu} Y$, Li Q, et al. Construction and characterization of SPI-2 deletion mutant of Salmonella Pullorum S06004. Wei Sheng Wu Xue Bao. 2015; 10.13343/j.cnki.wsxb.20140621.

19. Haneda T, Okada N, Kikuchi Y, Takagi M, Kurotaki T, Miki T, et al. Evaluation of Salmonella enterica serovar Typhimurium and Choleraesuis slyA mutant strains for use in live attenuated oral vaccines. Comp Immunol Microbiol. 2011;34:399-409.

20. Rana N, Kulshreshtha RC. Cell-mediated and humoral immune responses to a virulent plasmid-cured mutant strain of Salmonella enterica serotype gallinarum in broiler chickens. Vet Microbiol. 2006;115:156-62.

21. Song $H$, Yan $R, X u L$, Song $X$, Shah MA, Zhu H, et al. Efficacy of DNA vaccines carrying Eimeria acervulina lactate dehydrogenase antigen gene against coccidiosis. Exp Parasitol. 2010;126:224-31. 
22. Cirillo DM, Valdivia RH, Monack DM, Falkow S. Macrophage-dependent induction of the Salmonella pathogenicity island 2 type III secretion system and its role in intracellular survival. Mol Microbiol. 1998;30:175-88.

23. Collins FM, Scott MT. Effect of Corynebacterium parvum treatment on the growth of Salmonella enteritidis in mice. Infect Immun. 1974;9:863-9.

24. Matsuda K, Chaudhari AA, Lee JH. Evaluation of safety and protection efficacy on cpxR and lon deleted mutant of Salmonella Gallinarum as a live vaccine candidate for fowl typhoid. Vaccine. 2011;29:668-74.

25. Wigley $P$, Jones MA, Barrow PA. Salmonella enterica serovar Pullorum requires the Salmonella pathogenicity island 2 type III secretion system for virulence and carriage in the chicken. Avian Pathol. 2002;31:501-6.

26. Heithoff DM, House JK, Thomson PC, Mahan MJ. Development of a Salmonella cross-protective vaccine for food animal production systems. Vaccine. 2015;33:100-7

27. Nandre RM, Lee D, Lee JH. Cross-protection against Salmonella Typhimurium infection conferred by a live attenuated Salmonella Enteritidis vaccine. Can J Vet Res. 2015;79:16-21

28. Yin J, Cheng Z, Wang X, Xu L, Li Q, Geng S, et al. Evaluation of Salmonella enterica serovar Pullorum pathogenicity island 2 mutant as a candidate live attenuated oral vaccine. Clin Vaccine Immunol. 2015;22:706-10.

\section{Submit your next manuscript to BioMed Central and take full advantage of:}

- Convenient online submission

- Thorough peer review

- No space constraints or color figure charges

- Immediate publication on acceptance

- Inclusion in PubMed, CAS, Scopus and Google Scholar

- Research which is freely available for redistribution 